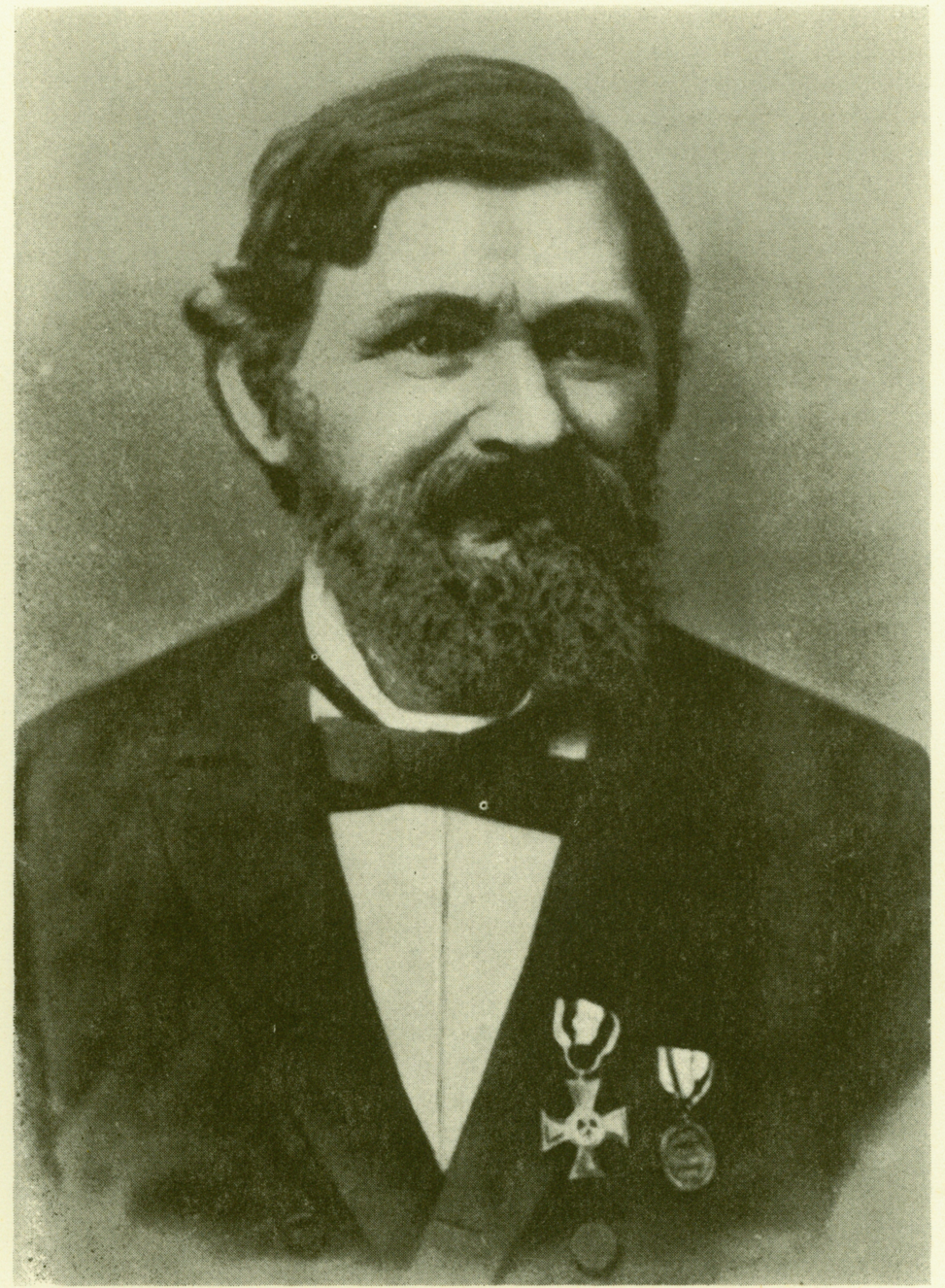

D. F. E. MEISSEL 1890 


\section{Hilbert's Double Series Theorem and Principal Latent Roots of the Resulting Matrix}

The inequality $\sum_{m=1}^{\infty} \sum_{n=1}^{\infty} \frac{a_{m} a_{n}}{m+n-1} \leqq \pi \sum_{p} a_{p}{ }^{2}$, was proved by HILBERT and published by WEYL. ${ }^{1}$ Various proofs were given by HARDY, LITTLEWOOD \& Pólya. ${ }^{2}$ In this inequality $\pi$ is the best possible constant; that is, the maximum value of $\sum_{m} \sum_{n} \frac{a_{m} a_{n}}{m+n-1} / \sum_{p} a_{p}{ }^{2}$ for arbitrary $\left\{a_{p}\right\}$ is $\pi$. It is no longer the best possible sum when the summation is finite; from 1 to $N$, say. In this case FRAZER ${ }^{3}$ has shown that $(N+1) \sin [\pi /(N+1)]$ is better. But this result is not the best possible, and Copsey, Frazer, \& Sawyer have published investigations ${ }^{4}$ based on empirical values of the constant $\lambda$ for $N=1(1) 5,10,20$, computed by the Royal Aircraft Establishment. Further computations for $N=2(1) 20$ are being made by the National Physical Laboratory.

The ordinary method for maximizing this quadratic form shows ${ }^{5}$ that the best possible value of the constant is the greatest latent root of the matrix $\|1 /(m+n-1)\| ; n \leqq N$, and it was in this way that the values were computed.

The roots and vectors of the segments of the Hilbert matrices of $N$ rows were derived from the iterated multiplication of the matrix into an arbitrary column vector, the procedure and accelerating processes being essentially those of Aitken. ${ }^{6}$ Operations were carried out on British Hollerith machines following the cycle, tabulator-reproducer-sorter-multiplier-tabulator. Machines were checked in the usual way by check sums. Final checking was done on a Brunsviga 20 machine, and roots and vectors for $n=6$ and 8 were added at this stage.

An approximate relation between $N$ and $\lambda$ of the form $1 /(\pi-\lambda)$ $=a \ln (N+b)+c$ has been found, ${ }^{4}$ but it is evidently incomplete as the error increases rapidly with $N$. Dr. Olga ToDD, in a recent unpublished communication, has shown that though $\lambda$ tends to $\pi$ asymptotically as $N$ tends to $\infty, \pi$ is not a latent root of the infinite matrix. Further, she shows that $\lambda=\pi\{1+\mathrm{O}(1 / \log N)\}$ and $\sum_{1}^{N} \sum_{1}^{N} \frac{a_{m} a_{n}}{m+n-1} / \sum_{1}^{N} a_{p}{ }^{2} \sim \pi\{1+\mathrm{O}(1 / \log N)\}$, when $\left\{a_{p}\right\}=\{1,1 / \sqrt{2}, 1 / \sqrt{3}, 1 / \sqrt{4}, \cdots 1 / \sqrt{N}\}$.

Principal Latent Root and Vector of Segments of the Hilbert Matrix

\begin{tabular}{|c|c|c|c|c|c|}
\hline $\mathbf{N}$ & 2 & 3 & 4 & 10 & 20 \\
\hline Latent Root & 1.26759188 & 1.40831893 & 1.50021428 & 1.75191967 & 1.90713472 \\
\hline Latent Vector & & & & & \\
\hline & 0.53518376 & 0.55603256 & 0.57017208 & 0.60899191 & 0.63153893 \\
\hline & & 0.39090795 & 0.40677899 & 0.45313830 & 0.48170552 \\
\hline & & & 0.31814097 & 0.36528601 & 0.39577939 \\
\hline & & & & 0.30775305 & 0.33864052 \\
\hline & & & & 0.26672518 & 0.29732839 \\
\hline & & & & 0.23580131 & 0.26579806 \\
\hline & & & & 0.21156396 & 0.24080108 \\
\hline & & & & 0.19200513 & 0.22041627 \\
\hline & & & & 0.17586003 & 0.20342569 \\
\hline & & & & & 0.18901 \\
\hline
\end{tabular}


Latent Vector

$\begin{array}{ll}1 & \\ 0.58056 & 692 \\ 0.41880 & 095 \\ 0.33006 & 105 \\ 0.27325 & 824\end{array}$

1 0.58862854 0.42832928 0.33966189

0.28252359 0.24233781
1

0.60050425

0.44267155

0.35437045

0.29691858

0.25618093

0.22562937

0.20179019

\section{R. A. FAIRThorne}

Royal Aircraft Establishment South Farnborough, England

\section{J. C. P. Miller}

${ }^{1} \mathrm{H}$. WEYL, Singuläre Integralgleichungen mit besonderer Berücksichtigung des Fourierschen Integraltheorems. Diss. Göttingen, 1908.

2 G. H. Hardy, J. E. Litrlewood, \& G. Pólya, Inequalities, Cambridge, 1934, p. 226-259. p. 7-9.

${ }^{3}$ H. Frazer, "Note on Hilbert's inequality," London Math. Soc., Jn., v. 21, 1946,

"E. H. Copsey, H. Frazer, \& W. W. Sawyer, "Empirical data on Hilbert's inequality," Nature, v. 161, 6 Mar. 1948, p. 361.

${ }^{5} \mathrm{R}$. Courant \& D. Hilbert, Methoden der mathem. Physik, second ed., v. 1, Berlin, 1931 ; U.S.A. photo-lithoprint, 1943.

'A. C. Artken, "Studies in practical mathematics. II. The evaluation of the latent roots and latent vectors of a matrix," R. Soc. Edinb., Proc., v. 57, p. 269-304, 1937.

\section{Piecewise Polynomial Approximation for Large-Scale Digital Calculators}

1. Introduction. Most large-scale digital calculating machines are equipped to perform automatically the arithmetic operations of addition, subtraction, multiplication, division, and in some cases of extracting the square root. All arithmetic processes must be carried out by suitably combining these given operations. But many functions whose evaluation is frequently required, such as the elementary transcendental functions, for example, cannot be represented exactly by any combination of a finite number of the given operations. In order to evaluate such functions, it is necessary to resort to some sort of approximation.

A method frequently employed may be called "piecewise polynomial approximation." This method consists of dividing the interval upon which the required function is to be approximated into a number of sub-intervals upon each of which the function is represented by a polynomial. The coefficients of these polynomials are stored within the machine or external to it in a manner consistent with the machine's construction. When the value of the independent variable is given, the proper sub-range is selected by the machine itself. The operations of addition and multiplication applied to the value of the independent variable and to the stored coefficients are then sufficient to evaluate the appropriate polynomial and hence to obtain an approximation to the required function.

In practice, the range over which the approximation is to hold and the maximum allowable error are usually known in advance. We shall assume that the maximum allowable degree of the approximating polynomials is 